\title{
Application of the Force Control on the Working Path Tacking
}

Sun-Run Wang

Professor, Department of Mechanical Engineering, National Central University, Chung-Li, Taiwan 32054, R.O.C.

Zhi-Zhong Qiao

Professor, Department of Mechanical Engineering, National Central University, Chung-Li, Taiwan 32054, R.O.C.

Pi-Cheng Tung

Professor, Department of Mechanical Engineering, National Central University, Chung-Li, Taiwan 32054, R.O.C., t331166@ncu.edu.tw

Follow this and additional works at: https://jmstt.ntou.edu.tw/journal

Part of the Mechanical Engineering Commons

\section{Recommended Citation}

Wang, Sun-Run; Qiao, Zhi-Zhong; and Tung, Pi-Cheng (2002) "Application of the Force Control on the Working Path Tacking," Journal of Marine Science and Technology. Vol. 10: Iss. 2, Article 4.

DOI: 10.51400/2709-6998.2307

Available at: https://jmstt.ntou.edu.tw/journal/vol10/iss2/4

This Research Article is brought to you for free and open access by Journal of Marine Science and Technology. It has been accepted for inclusion in Journal of Marine Science and Technology by an authorized editor of Journal of Marine Science and Technology. 


\title{
APPLICATION OF THE FORCE CONTROL ON THE WORKING PATH TRACKING
}

\author{
Sun-Run Wang, Zhi-Zhong Qiao, and Pi-Cheng Tung*
}

Key words: compliance control, manipulator, robot, self-tuning, path tracking, radius compensation, fuzzy control.

\begin{abstract}
For the application of tracking the path on workpieces, the CCD (Charge Couple Device) identificating system is generally used as one of the popular resolutions. However, the implementation of CCD identificating system is complex and environmental-limited. This study presents a compliant motion controller by using the end-effector installed with a force sensor. This results the smoothly path tracking on a workpiece. By applying the algorithm of Fuzzy into this compliant motion, the compliant control is improved and the path on the workpiece is found more quickly and accurately. A radius compensating method is used to correct the measured data. The experiments performed have successfully demonstrated the feasibility of the proposed control algorithm.
\end{abstract}

\section{INTRODUCTION}

The task under consideration is to control the manipulator and let the end-effector follow an unknown path on a workpiece. This can be used to weld the workpieces together, cut the workpiece into two or more pieces and so on. To implement the task of tracking along an unknown path, the control system must rely on the reliable data collected by the sensors. The equipments used in this experiment include two kinds of sensors. They are encoders built on each of the five joints of the manipulator and a force sensor mounted on the wrist of the manipulator. The encoder on each joint of the manipulator can sense the position of the endeffector, and receive the command from its driver to move the end-effector with controllable manner. The force sensor has been used to maintain in contact with the workpiece and determine the tangent plane of the contact plane to create the direction of working path. In this study, we ignore the friction force at the contact point between the tool grasped by the end-effector and

Paper Submitted 03/30/02, Accepted 05/17/02. Author for Correspondence: Pi-Cheng Tung.

*Professor, Department of Mechanical Engineering, National Central University, Chung-Li, Taiwan 32054,R.O.C.E-mail: t331166@ncu.edu.tw the workpiece.

The manipulator of contact measurement type is extensively used in recent years. Two kinds of methods for performing compliant motion of this manipulator were developed. The development of compliant motion can be categorized as either hybrid force/position control [1] or impedance control [2, 3, 4]. The first approach attempts to control the force and position separately, while the second one concerned the relationship between the contact forces and manipulator position. Since the dynamic model for the contact force interaction system is unknown, these two methods are not applicable. This mathematical model must be nonlinear, time dependent and highly coupled. To model, identify and control such a complex system is by no means an easy task. The fuzzy controller can be used to solve this problem. Thus, several self-tuning fuzzy controllers capable of automatically implementing the fuzzy rules have been proposed and extensively studied $[5,6,7]$. F. Y. Hsu and L. C. Fu [8] designed a new hybrid force/ position controller with adaptive fuzzy approach for the robot manipulators. Their approaches are successfully applied to the deburring process. For unknown paths or contours, all controllers for compliant motions fail, since the desired trajectory cannot be obtained. This study, however, adopts the fuzzy control method to implement the on-line identification about the environment and response effectively. An effective compliant controller is developed without knowing the desired path in advance. Moreover, the force/torque sensors and fuzzy logic controllers with active compliance control are used to control the contact force between the end-effector and the workpiece.

The following sections will demonstrate the dynamic model of the robot manipulator, describe the design of the fuzzy controller, show the experimental results and end with the conclusion.

\section{DYNAMIC MODEL OF THE ROBOT MANIPULATOR}

Consider the robot manipulator whose tool will 


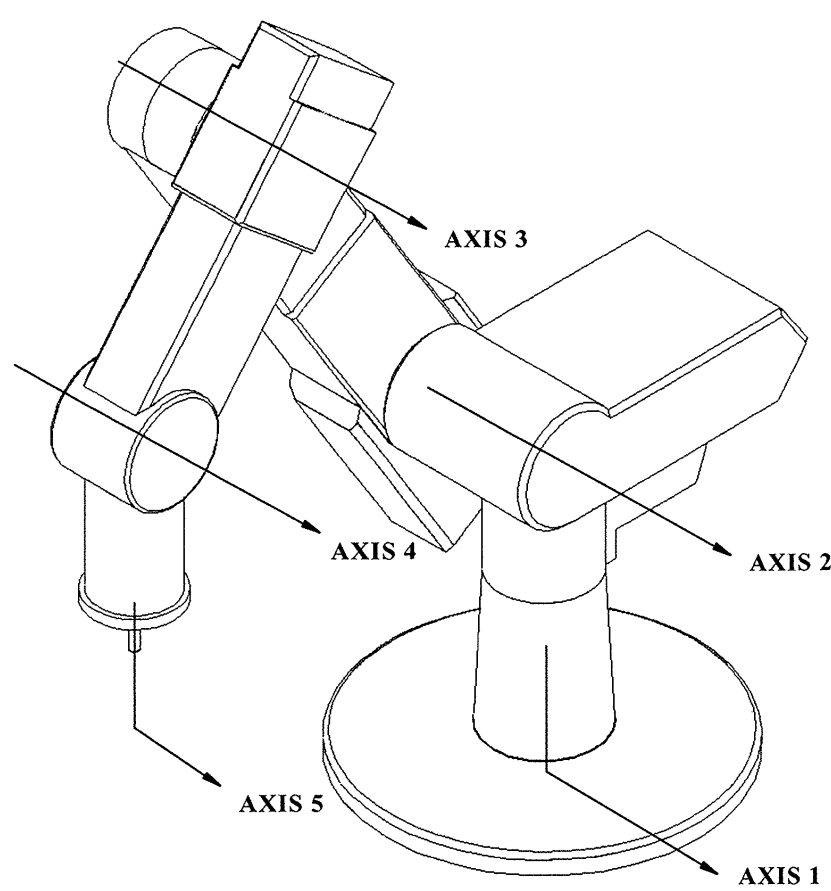

Fig. 1. Five degrees of freedom SCORBOT-ER VII robot.

perform path-tracking motion, as shown in Figure 1. According to [10], the dynamic model of this robot manipulator in joint space can be derived as

$$
M(q) \ddot{q}+C(q, \dot{q})+P(q)=\tau+\tau_{f},
$$

where $q, \dot{q}, \ddot{q} \in \mathfrak{R}^{6}$ are joint angle vector, joint velocity vector and joint acceleration vector, respectively. $M(q)$ $\in \mathfrak{R}^{6 x 6}$ is the inertia matrix of the robot, $C(q, \dot{q})$ denotes the coriolis and centrifugal torques, $P(q)$ stands for the term caused by gravity, $\tau_{f} \in \mathfrak{R}^{6}$ is the joint torque vector, and $\tau_{f} \in \mathfrak{R}^{6}$ represents the constrained torque vector generated on the joints by the environment. To simplify the controller design for the path-tracking task, the space of this dynamic model will be transformed into the Cartesian coordinates. By assuming the Cartesian coordinates of the end-effector $x$ can be expressed as a function of the joint coordinates $q$, that is

$$
x=D(q),
$$

where $x=\left[x_{1}, x_{2}, \ldots x_{6}\right]^{T} \in \mathfrak{R}^{6}$, the Equation (2) can be differentiated to get

$$
\begin{aligned}
& \dot{x}=\frac{\partial D(q)}{\partial q} \dot{q}=J(q) \dot{q}, \\
& \ddot{x}=J(q) \ddot{q}+\dot{J}(q) \dot{q},
\end{aligned}
$$

where $J(q) \in \mathfrak{R}^{6}$ is a Jocabin matrix of full rank for $q$.
There must exist a properly defined one-to-one mapping between $x$ and $q$. Let the inverse matrix of $J$ be denoted as $J^{-1}$. Then we get

$$
\begin{aligned}
& M_{x}=J^{-1 T} M J^{-1} \\
& C_{x}=J^{-1 T} C J^{-1}-J^{-1 T} M J^{-1} \dot{J} J^{-1} \\
& P_{x}=J^{-1} P \\
& f_{e}=J^{-1 T} \tau_{f} \\
& f=J^{-1 T} \tau .
\end{aligned}
$$

The dynamic equations of the robot manipulator in the Cartesian frame can be represented as

$$
M_{x}(x) \ddot{x}+C_{x}(x, \dot{x})+P_{x}(x)=f+f_{e} .
$$

In the path tracking process, the tangential directions to the path can be derived from the measured force. Without previous knowledge of the path on the workpiece, the manipulator can precisely comply with the curved path.

\section{DESIGN OF THE FUZZY CONTROLLER}

The concept of Fuzzy sets was brought up by American Zadeh at 1965. After then, the Fuzzy Mathematics is popularly applied into the control system. To design a Fuzzy controller, it is not necessary to understand the mathematics model of the system. The knowledge base will be created by a set of control rules instead. According to these control rules, the control performance of the system must be improved.

In this force control system, we assume that there is no any frictional force between the end-effector and the workpiece, and both of them are taken as the rigid bodies. The equations of the system can then be written as:

$$
F=k X
$$

where $F$ is the contact force, $k$ is the displacement of the end-effector, and is the coefficient of elasticity.

During the processes of path-tracking implemented by the robot, there exists an optimal value for each displacement. The force control requires the robot to move faster and larger before it contacts with the workpiece. In order to hold the accuracy of the contact force, the displacement of the robot should be slowed down when the end-effector contacts with the workpiece. In this research, we define the displacement of the robot as $\Delta x$, and tune the parameters by the fuzzy controller. 
The requirements of the velocity and accuracy will be satisfied accordingly with the fuzzy controller applied.

Figure 2 shows the control system with the fuzzy controller. In this research, the parameter $\Delta x$ is determined by the error of the force, $e(k)$. That is

$$
\text { If } e(k) \text { is } A_{i} \text {, then } \Delta x \text { is } B_{i}, i=1,2, \ldots, m \text {. }
$$

where $A_{i}$ is the fuzzy set on the corresponding supporting sets. The membership functions (MF) of these fuzzy sets for $e(k)$ are shown in Figure 3. The fuzzy set $B_{i}$ is characterized by the membership functions shown in Figure 4.

A set of rules is shown in Table 1. The defuzzification processes are implemented by the Center of Gravity method.

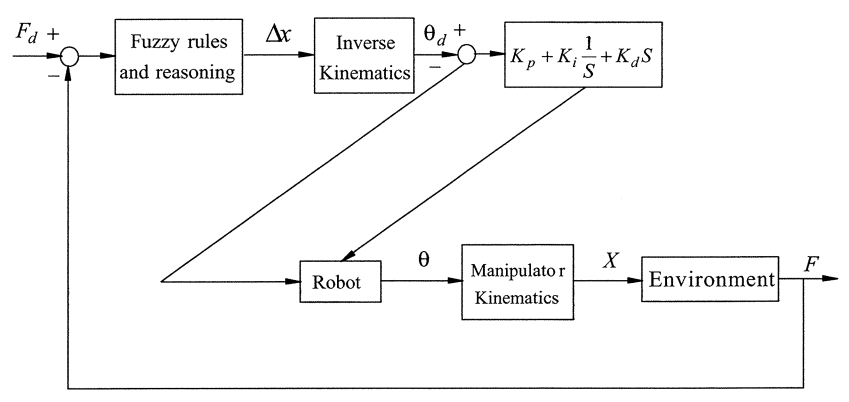

Fig. 2. Block diagram of the fuzzy controller.

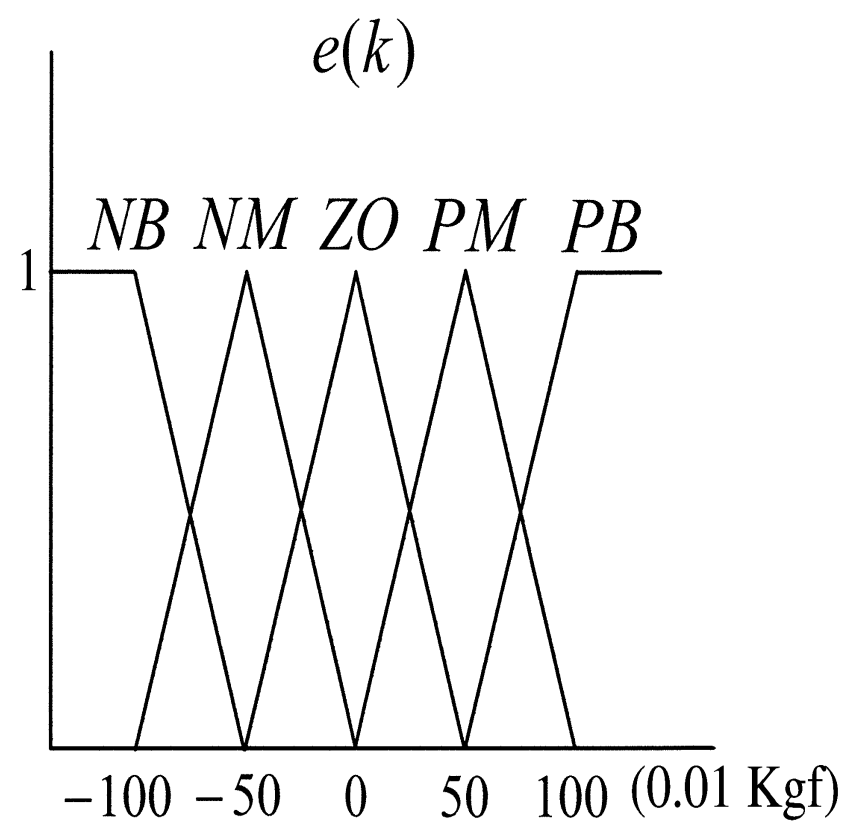

Fig. 3. The membership functions of the error.

$$
y^{*}=\frac{\sum_{l=1}^{m} \int_{Y} y B_{l}(y) d y}{\sum_{l=1}^{m}{ }_{Y} B_{l}(y) d y}
$$

where $B(y)$ is the fuzzy set.

\section{EXPERIMENTAL SETUP AND RESULTS}

The control system is schematically shown in Figure 5. The five axes robot SCORBBOT-ER VII that designed by ESHED ROBOTEC Company is used to perform this experiment. Five AC servomotors are used to drive the five axes of the manipulator. The optical encoders are installed on the manipulator. A force sensor NITTA UFS 3012A SN_N25 with a resolution of $0.01 \mathrm{~kg}$ and an amplifier NITTA UFS EE-161 are installed on the manipulator. The controllers of type PCL-818 and PCL-726 are designed by ADVANTECH Co. LTD. The position and force signals are sent to the servomotors from the personal computer through a D/A converter. The control program is coded by $\mathrm{C}$ language. The systematic notation for the right-handed orthogonal

Table 1. The Fuzzy rules of $\Delta x$

\begin{tabular}{c|ccccc}
\hline & \multicolumn{5}{|c}{$e(\mathrm{k})$} \\
\hline$\Delta x$ & $\mathrm{NB}$ & $\mathrm{NM}$ & $\mathrm{ZO}$ & $\mathrm{PM}$ & $\mathrm{PB}$ \\
& $\mathrm{B}$ & $\mathrm{M}$ & $\mathrm{S}$ & $\mathrm{M}$ & $\mathrm{B}$ \\
\hline
\end{tabular}

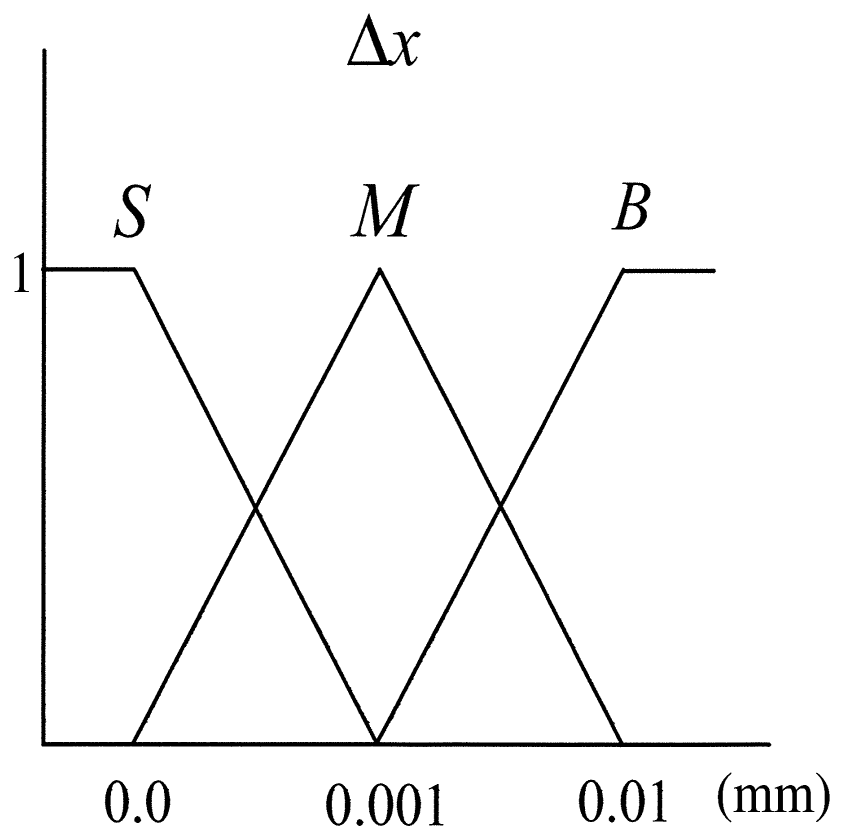

Fig. 4. The membership function of $\Delta x$. 
coordinate system is represented in Figure 6.

The measured data must be fitted before applying them to construct the real path, since there are some errors existing in measured data. In the measuring process, the errors of the force sensors and any other external disturbance may end up with unsuccessful movement of the end-effector. In this study, the radius compensating method is adopted to generate the differential signals and to magnify the errors of data. This magnification process will create many noises after radius compensation. To resolve this defect, we take some advantage of the hardware to design an easier and more efficient calculation method. This method, although not modifying the contents of the data, rejects most of the improper data. Moreover, this method functions exactly like a signal filter.

The $0.55 \mathrm{kgf}$ contact force seems to be appropriate in this experiment. The movement of the end-effector and the contact force are controlled and tuned by the fuzzy controller. Figure 7 displays the variations of contact force, one is for the end-effector moves with constant step, and the other is for the end-effector controlled by this self-tuning fuzzy controller. In Figure 8 , the inner path is the path of the end-effector, and

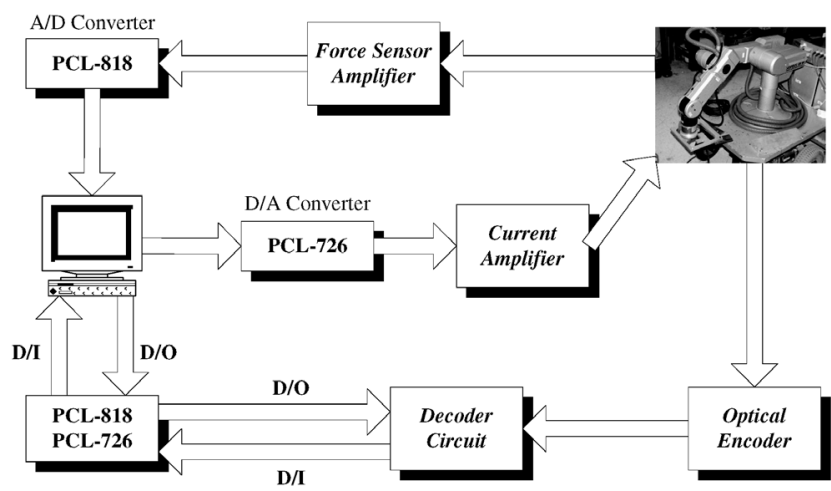

Fig. 5. The basic structure of this experiment.

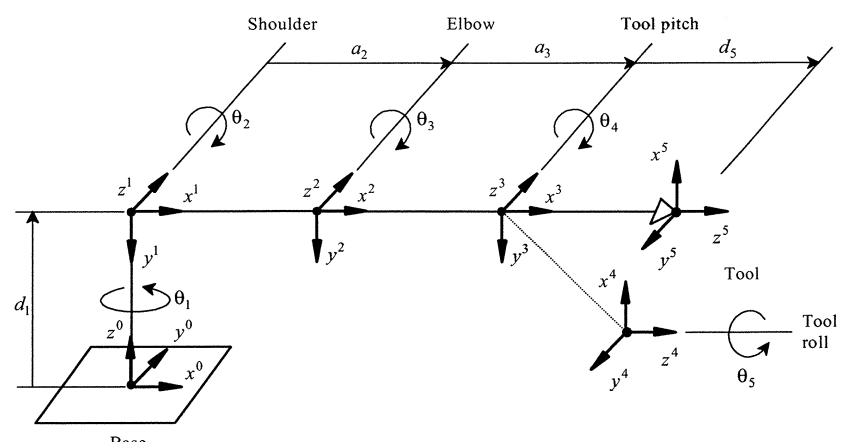

Fig. 6. Coordinates of the SCORBOT-ER VII robot. the outer path is the path on the workpiece obtained by radius compensation. The distance between the center of end-effector and the boundary of the workpiece is the radius of the end-effector, which is $5 \mathrm{~mm}$. By compensating this distance, we obtain the real path on the workpiece from the measured data. The accuracy will increase if the radius of the end-effector decreases.

Figure 9 to Figure 13 illustrates the variations of contact force for the positions A to E in Figure 8,

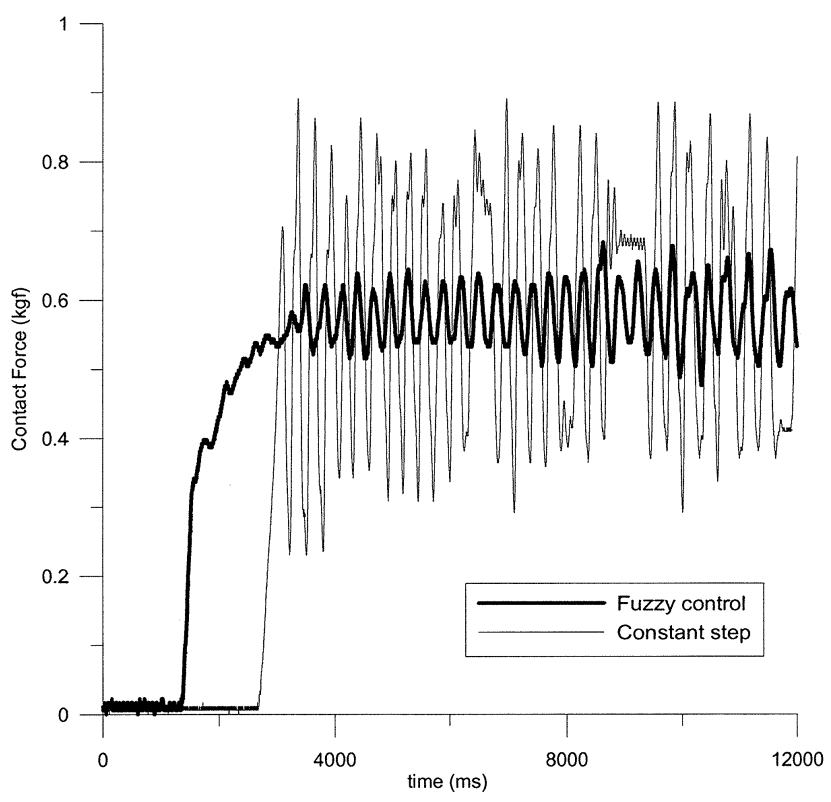

Fig. 7. The variation of contact force for fuzzy control vs. constant step.

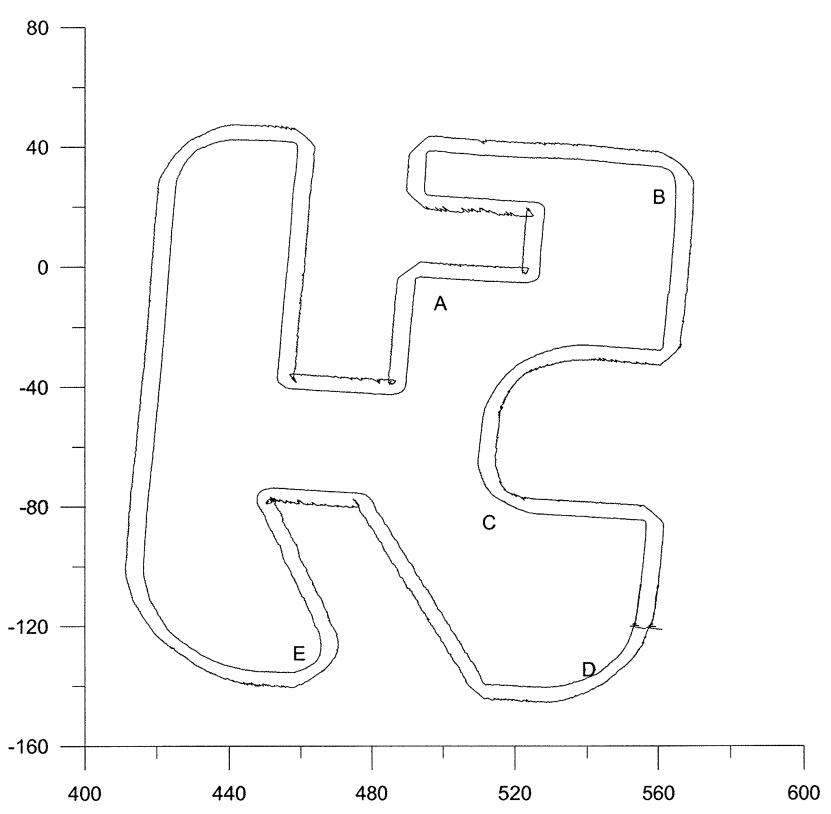

Fig. 8. The path on the workpiece. 


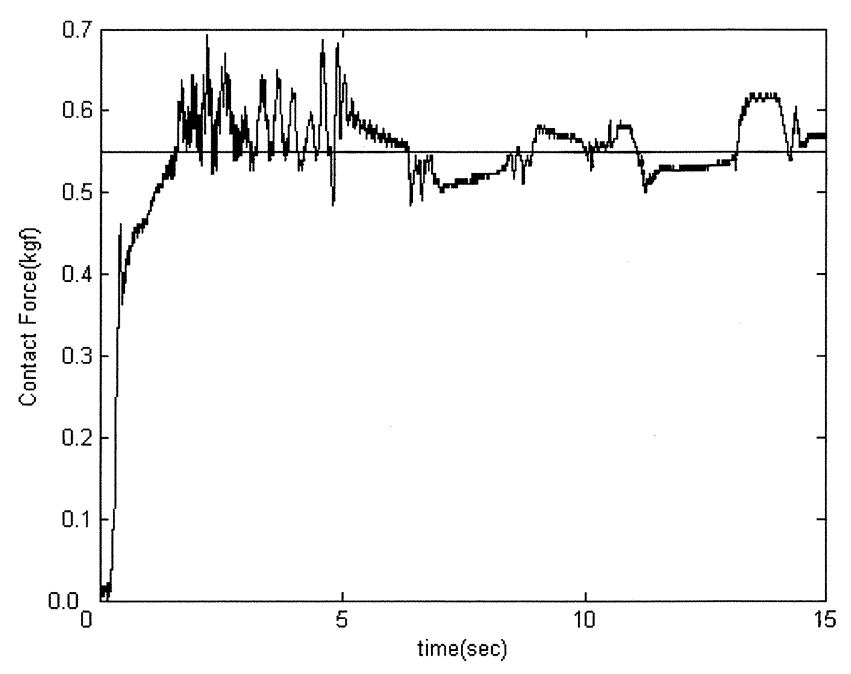

Fig. 9. The variation of the contact force at point A in Figure 8.

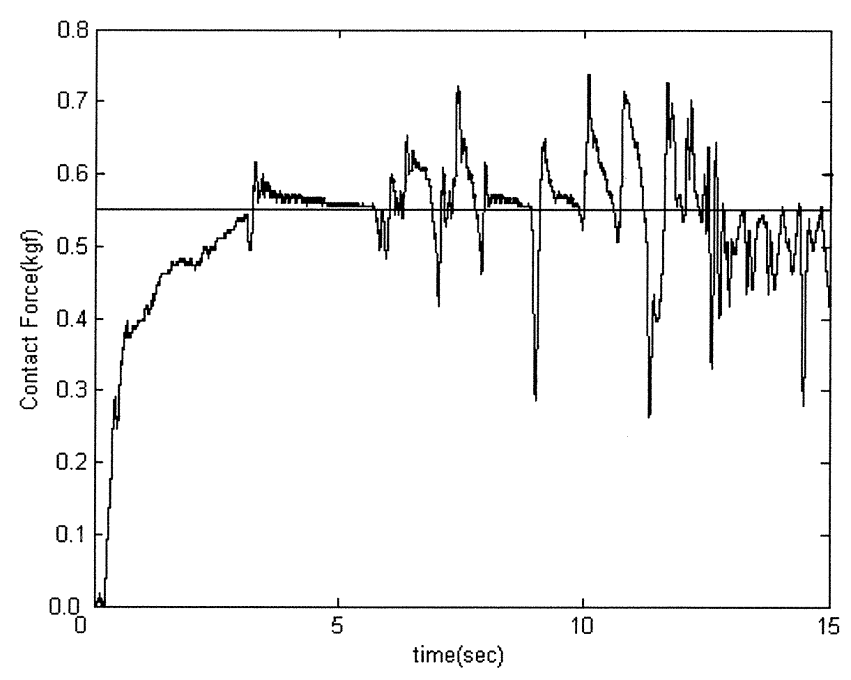

Fig. 10. The variation of the contact force at point B in Figure 8.

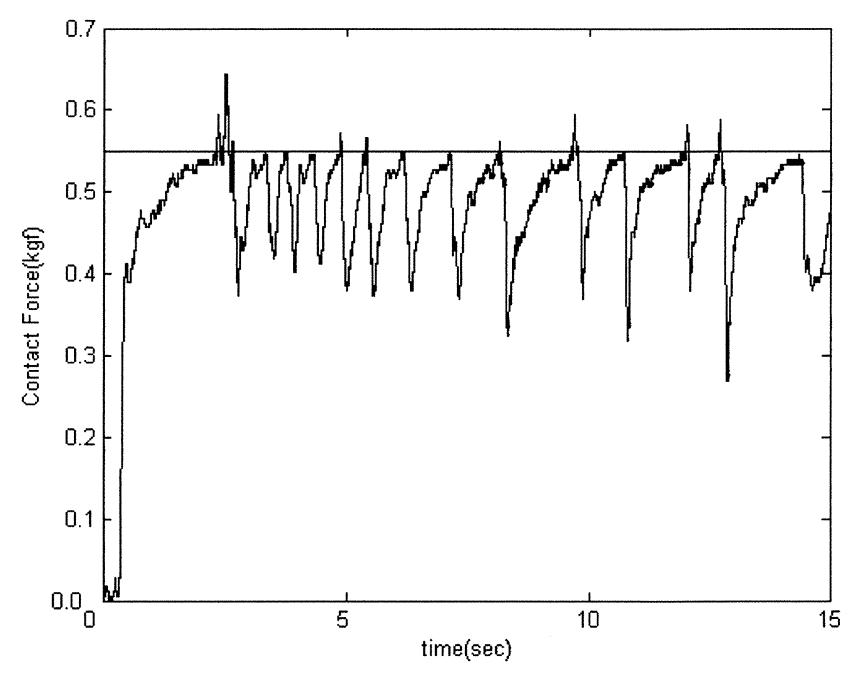

Fig. 11. The variation of the contact force at point $\mathrm{C}$ in Figure 8.

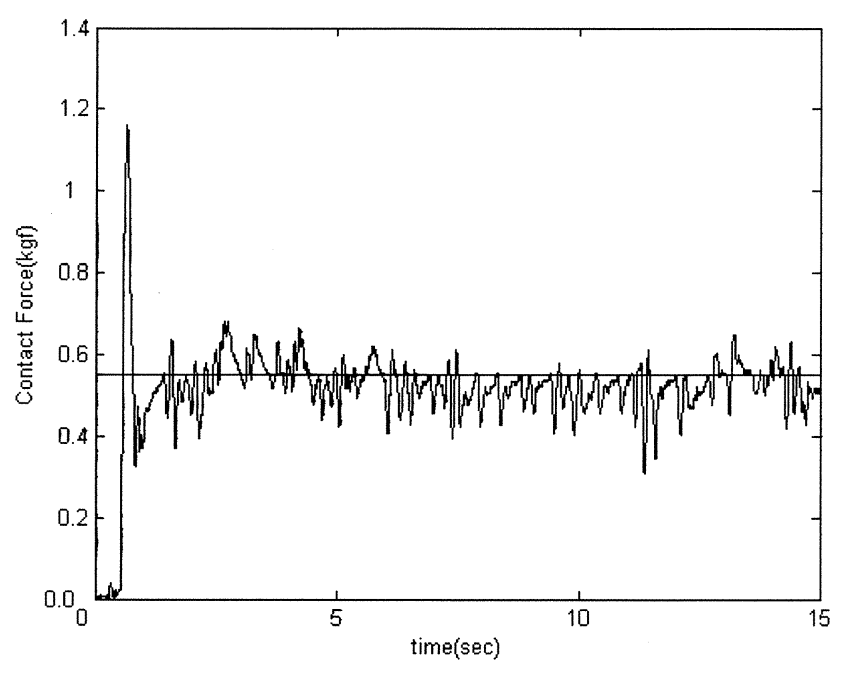

Fig. 12. The variation of the contact force at point D in Figure 8 .

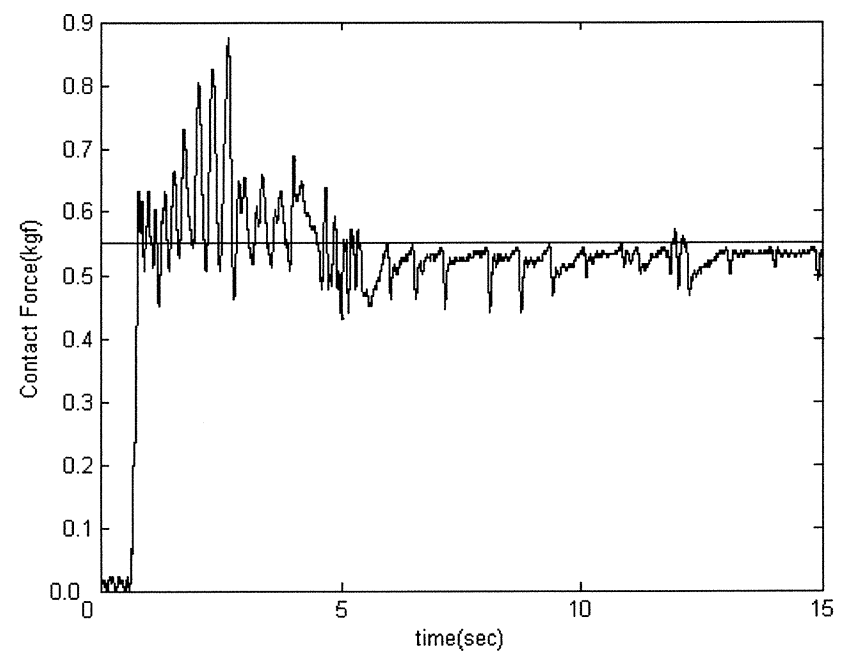

Fig. 13. The variation of the contact force at point $\mathrm{E}$ in Figure 8.

respectively. Position A is a concave angle of $90^{\circ}$, position $\mathrm{B}$ is a concave circle of radius $10 \mathrm{~mm}$. Position $\mathrm{C}$ is a convex circle of radius $15 \mathrm{~mm}$. Position D is concave circle of radius $30 \mathrm{~mm}$. Position $\mathrm{E}$ is a concave circle of radius $13 \mathrm{~mm}$.

\section{CONCLUSION}

This study presents a adaptive fuzzy control algorithm to implement automatic path tracking of the workpiece. The algorithm proposed herein also introduces the radius compensation method to match the experimental results. Although the measured paths of the workpieces near the sharp turning points still have some more disturbances, the self-tuning contact path 
tracking method adopted in this study can significantly reduce the noises. The measuring time varies with the different conditions automatically.

\section{REFERENCE}

1. Raibert, M.H. and Craig, J.J., "Hybrid position/force control of manipulators", Trans. ASME J. of Dyn. Systems, Measurement and Control, Vol. 102, pp. 126-133 (1981).

2. Hogan, N., "Impedance Control: An approach to Manipulation: Part I - Theory, Part II - Implementation, Part III - Application", Trans. ASME J. of Dyn. Systems, Measurement, and Control, Vol. 107, pp. 1-24 (1985).

3. Kazerooni, H., Waibel, B.J. and Kim, S., "On the stability of robot compliant motion control: theory and experiments", Trans. ASME J. of Dyn. Systems, Measurement, and Control, Vol. 112, pp. 417-426 (1990).

4. Hogan, N., "On the stability of manipulators performing contact tasks", IEEE Trans. on Robotics and Automation, Vol. 4, pp. 667-686 (1988).

5. He S.Z., Tan, S. and Hang, C.C., "Control of dynamical processes using an on-line rule-adaptive fuzzy control system”, Fuzzy Sets and Systems, Vol. 54, pp. 11-22 (1993).

6. Llama, M.A., Santibanez, V., Kelly, R. and Flores, J., "Stable fuzzy self-tuning computed-torque control of robot manipulators, Proceedings", IEEE Inter. Conf. On Robotics and Automation Part 3 (of 4) (1998).

7. Fan, K.C., "Non-contact automatic measurement for free-form surface profiles", Computer Integrated Manufacturing Systems, Vol. 10, No. 4, pp. 277-285 (1997).

8. Hsu, F.Y. and Fu, L.C., "Intelligent robot deburring using adaptive fuzzy hybrid position/force control", IEEE Transactions on Robotics and Automation, Vol. 16, No. 4 , pp. 325-335 (2000). 